$\$$ Research Square
Preprints are preliminary reports that have not undergone peer review.

They should not be considered conclusive, used to inform clinical practice, or referenced by the media as validated information.

\title{
Race/Ethnicity, Delay of Adjuvant Chemotherapy Initiation Following Breast Cancer Surgery and Associated Patient Survival: An Analysis of the United States National Cancer Database 2004-2016
}

\author{
Raj Piyush Desai \\ University of Virginia \\ Aditya Narayan \\ University of Virginia \\ Sabrina Hsiao \\ University of Virginia \\ Rajesh Balkrishnan ( $\square$ rbgws@yahoo.com ) \\ University of Virginia School of Medicine https://orcid.org/0000-0001-9363-9257
}

\section{Research Article}

Keywords: Breast Cancer, Treatment Delay, United States, Disparities, Ethnicity, Surgery

Posted Date: March 15th, 2021

DOI: https://doi.org/10.21203/rs.3.rs-289142/v1

License: (1) This work is licensed under a Creative Commons Attribution 4.0 International License. Read Full License 


\section{Abstract}

Introduction: The relationship between survival and time to the start of adjuvant chemotherapy (AC) among breast cancer patients is unclear. Most breast cancer patients start adjuvant chemotherapy within a few weeks after surgery, but it is still unclear whether a delay in the initiation of chemotherapy will lead to adverse outcomes.

Methods: Women diagnosed between 2004 and 2016 with invasive breast cancer (stages I-III) and treated with surgery and adjuvant chemotherapy were selected from the National Cancer Database $(n=443,100)$. We evaluated factors associated with prolonged time to start adjuvant chemotherapy ( $\geq 60, \geq 90$, and $\geq 120$ days after surgical resection) using multivariable log binomial models to estimate risk ratios (RRs) and $95 \%$ Cls. Cox proportional hazards model was used to investigate the effect of delay on survival.

Results: The average time to adjuvant chemotherapy was 47.93 days ( \pm 29.17 days). Non-Hispanic African American patients had higher risk of 60 day delay ( $\mathrm{RR}, 1.41 ; 95 \% \mathrm{Cl}, 1.38$ to 1.43 ), 90-day delay (RR, 1.55; 95\% Cl, 1.50 to 1.60), and 120-day delay (RR, $1.69 ; 95 \% \mathrm{Cl}, 1.61$ to 1.78$)$ compared with non-Hispanic white patients. Early initiation of chemotherapy, less than 1 month (RR, 0.893; 95\% Cl, 0.86 to 0.92 ), 1-2 month (RR, 0.80; $95 \% \mathrm{Cl}$, 0.77 to 0.82 ), and $2-3$ month (RR, $0.87 ; 95 \% \mathrm{Cl}, 0.84$ to 0.90 ); was associated with decreased overall mortality. Non-Hispanic African Americans (RR, $1.16 ; 95 \% \mathrm{Cl}, 1.13$ to 1.19$)$ were associated with increased overall mortality compared to non-Hispanic whites.

Conclusion: Black breast cancer patients experience clinically relevant delays in the initiation of adjuvant chemotherapy more often than white patients, which may in part explain the increased mortality observed among black patients. Efforts should be made to reduce the time to surgery when possible to enhance overall survival.

\section{Introduction}

Breast cancer is among the highest mortality forms of cancer and is the most common form among women [1]. After lung cancer, breast cancer is the second leading cause of death among women in the United States [2]. The prevalence of breast cancer is increasing due to the population's growing exposure to a range of risk factors, including changes in the environment, healthcare access, and patient lifestyle. The lifetime risk of developing breast cancer is currently $12.4 \%$ in women $[3,4,5]$ In 2018 over 2 million new cases were diagnosed, representing $23 \%$ of all cancers. It is now the most common cancer in developed and developing regions, though incidence varies widely with race and ethnicity [6, 7].

There is a wide array of treatment options for breast cancer. The preferred approach largely depends on the severity, malignancy, and other properties of the cancer given the disease's multifactorial nature. As with many cancers, the most common approaches are surgery, radiation therapy, chemotherapy, hormone therapy, and immunotherapy. Breast cancer consists of three subtypes based on estrogen/progesterone receptor expression and ERBB2 gene amplification, each with unique risk profiles and treatment approaches. For non-metastatic breast cancers, tumor resection (lumpectomy), followed by mastectomy and axillary lymph node surgery are coupled with systemic neoadjuvant or adjuvant therapies (preoperative and postoperative respectively) [8]. Nearly $90 \%$ of women will have an early-stage form of the disease, which presents with particularly high relapse risk. This finding is based on estimates from Mariotto et al. of roughly 150,000 women in the US living with metastatic breast cancer in 2017 [9]. Micrometastases have also shown an impact on long-term outcomes following a breast cancer diagnosis. Maibenco et al. demonstrated that 12-year survival was reduced among women with either solitary or multiple lymph nodes with micrometastases relative to cancer that has not spread to the lymph nodes (node-negative) [10]. Boer et al. found that isolated tumor cells or micrometastases in regional lymph nodes leads to reduced 5-year disease-free survival rate in women with favorable early-stage breast cancer without adjuvant therapy as well as significant increases in disease-free survival on receipt of adjuvant therapy [11]. Finally, Andersson et al. found that ten-year cancer-specific survival and overall survival were lower in cases of micrometastases relative to node-negative cases [12].

Adjuvant treatment (treatment after surgery) is the primary mechanism of combating cancer relapse as well as metastases and involves radiation (local), cytotoxic chemotherapy (systemic), or endocrine approaches. Adjuvant therapy has led to decreased mortality, morbidity, and prevalence of breast cancer (30-40\% based on the Early Breast Cancer Trialists' Collaborative Group) [13]. Despite this demonstrated efficacy, there is minimal information regarding how the timing of administration impacts treatment outcomes [13, 14]. A recent meta-analysis by Zhan et al. found that a 4week delay before adjuvant chemotherapy administration significantly decreased overall and disease-free survival [15]. Similarly, Biagi et al. performed a systematic review that found that a 4-week delay in adjuvant chemotherapy resulted in a significant decrease in overall and diseasefree survival [16]. Several analogous studies have been performed for other types of cancer and stages of cancer, including late-stage colon cancer, early-stage breast cancer, gastric cancer, and pancreatic cancer [17-35]. The National Quality Forum, given such data, endorses chemotherapy initiation within 120 days of diagnosis or 90 days of surgery [36]. However, a number of studies describe a negligible difference: that delays in adjuvant therapy administration do not offer significant differences in treatment outcomes [37-40]. Collectively, these insights speak to the need for up-to-date, more conclusive data regarding treatment delays such that guidelines may be tailored to maximize survival rates for the highest-risk populations.

In considering risk factors, treatment delays have been shown to fall disproportionately along demographic lines. Gwyn et al. found potentially clinically significant differences in diagnosis and treatment delays between African American and white women between the ages of 20 and 54 [41]. Regarding adjuvant therapy administration specifically, over 2004-2006, Fedewa et al. found that most women received adjuvant chemotherapy 
within 60 and 90 days of surgery (85.2\% and 95.8\%, respectively). However, African American and Hispanic patients were found to have a higher risk of delay at both time points compared to white patients - though the extent of these delays was variable, and further analysis was required to understand this variability [42]. Gorin et al. corroborated these results as they identified that the greatest diagnostic, treatment and clinical delays for breast cancer were identified for African American women among other demographics [43]. Ultimately, the relationship between survival and time to the start of adjuvant chemotherapy (AC) among breast cancer patients is unclear. Most breast cancer patients start adjuvant chemotherapy within a few weeks after surgery. However, it is still unclear whether a delay in the initiation of chemotherapy will lead to adverse outcomes and what mediating variables influence the relationship between AC and treatment outcomes.

\section{Patients And Methods}

Data was gathered from the National Cancer Database (NCDB), a cancer registry based on hospital care which is sponsored by the ACS and American College of Surgeons. The NCDB includes standardized data on patient insurance state, tumor characteristics, course of treatment, outcomes, demographics, socioeconomic data, and treatment facility data. The database includes roughly $70 \%$ of newly diagnosed cancer cases in the U.S. and contains over 34 million records across over 1500 facilities. The data is collected using CoC (Commission on Cancer) program registries and is coded using their Facility Oncology Registry Data Standards. Given that no hospital, provider, patient identifiers, or protected health information was used in this study, institutional review board approval was not required. Along with the NCDB'S classifications, The Charlson-Deyo Index Score is a weighted derived from the sum of scores for a list of conditions in the Charlson Comorbidity Score Mapping Table based on the ICD-9-CM (International Class of Disease, 9th edition, Clinical Modification). This was used to assess comorbidity.

\section{Study Population}

The patient population consisted of women diagnosed with invasive breast cancer (stages I-III) between January 1, 2004 and December 31 st, 2016 from within the National Cancer Database $(n=432,883)$. Our analysis was restricted to women older than the age of 18.

In this study, we characterized four levels of adjuvant chemotherapy delay: Less than 1 month, 1-2 months, 2-3 months, and greater than 3 months. The time was defined based on the number of days following surgical resection to the start of adjuvant chemotherapy. In addition, the year of diagnosis was reported. Age was stratified into five groups: $18-49,50-59,60-69,70-79$, and over 80 . Patient race was characterized as nonHispanic White or non-Hispanic Black. The primary payer or insurance type held by the patient were classified by Medicaid, Medicare for patients between the ages of 18-64, Medicare for patients 65 and older, private health insurance (preferred provider organizations and health maintenance organizations), no insurance (self-pay, charity write-off, or those who are uninsured), and those with other or unknown data regarding insurance. Of note is the distinction between Medicare patients above the age of 65 and below the age of 65 , as the latter is limited to those with permanent disabilities. Regions were categorized by the US Census Bureau Regions and Divisions, which divide the country into 9 different divisions: New England, Middle Atlantic, South Atlantic, East North Central, East South Central, West North Central, West South Central, Mountain, and Pacific. The regions were further classified into four regions: Northeast- Middle Atlantic, New England; Midwest- West North Central, East North Central; WestPacific, Mountain; South- East South Central, West South Central, South Atlantic. The American Joint Commission on Cancer (AJCC) cancer stage was also included (Stage I, II or III). Based on the registry reporting of pre-existing comorbidities drawn from hospital discharge reports, the CharlsonDeyo comorbidity index was reported. Also related to the specific health status of the patient, their hormone receptor status (positive/borderline, negative, or no test available) was included in the analysis.

Additional social determinants of health were included in the collected patient data. Patient income was grouped into less than $\$ 38,000$, $\$ 38,000-\$ 47,999, \$ 48,000-\$ 62,999$, and $\$ 63,000+$. Further, the proportion of the population in a particular ZIP Code, based on prior census data, which did not attain a high school diploma was included in the collected data. Patients were grouped according to $21 \%$ or $\mathrm{more}, 13 \%-20 \%, 7 \%-12 \%$, and less than $7 \%$. Finally, given the issues faced by geographic barriers to accessing care, the data includes information on the patient's distance from the reporting facility.

\section{Statistical Analysis}

Women diagnosed between 2004 and 2016 with invasive breast cancer (stages I to III) who were treated with surgery and adjuvant chemotherapy were selected from the national cancer database. Descriptive statistics for and bivariate associations between groups were reported. We evaluated factors associated with prolonged time to start adjuvant chemotherapy ( $\geq 60, \geq 90$, and $\geq 120$ days after surgical resection) using multivariable log binomial models to estimate risk ratios and associated confidence intervals. Cox proportional hazards model was used to investigate the effect of delay on survival. All tests were two sided and a p-value of $<0.05$ was considered significant. The statistical analysis was performed using SAS version 9.4 (SAS Institute, Cary NC).

\section{Results}

The demographics and clinical characteristics of the study cohort are summarized in Table 1. There were significant differences across different racial groups with respect to delay of adjuvant chemotherapy. The average number of days from surgery to initiation chemotherapy was 48.73 days ( \pm 29.56 days) among all patients. Black patients had an average of 53.92 days, whereas Nonhispanic Whites averaged 47.38 days. Black patients 
were found to have the highest risk of mortality in the 120 day delay group ([HR], 1.69; $95 \% \mathrm{Cl},(1.61-1.78)$. Medicaid usage was more profound in non-hispanic Black communities (13.86\%) than whites (5.10\%), and it was highest in the Hispanic communities. There was a notable difference in regions between Nonhispanic Whites and Blacks for the South and West regions. The highest Charlson Deyo Score was found for black patients in comparison to the other five groups.

Table 1. Patient Characteristics by Race, NCDB, 2004-2016 ( $\mathrm{n}=432,883)$ 


\begin{tabular}{|c|c|c|c|c|c|c|c|c|c|c|c|c|c|}
\hline \multirow[t]{2}{*}{ Characteristic } & \multicolumn{2}{|c|}{$\begin{array}{l}\text { Total } \\
(n=432,883)\end{array}$} & \multicolumn{2}{|c|}{$\begin{array}{l}\text { White } \\
(n=333,713)\end{array}$} & \multicolumn{2}{|c|}{$\begin{array}{l}\text { Hispanic } \\
(n=24,657)\end{array}$} & \multicolumn{2}{|c|}{$\begin{array}{l}\text { Black } \\
(n=57,020)\end{array}$} & \multicolumn{2}{|c|}{$\begin{array}{l}\text { Asian } \\
(n=13,428)\end{array}$} & \multicolumn{2}{|c|}{$\begin{array}{l}\text { Other/Unknown } \\
(n=4,065)\end{array}$} & \multirow[t]{2}{*}{$P$} \\
\hline & $\mathrm{N}$ & $\%$ & $\mathrm{~N}$ & $\%$ & $\mathrm{~N}$ & $\%$ & $\mathrm{~N}$ & $\%$ & $\mathrm{~N}$ & $\%$ & $\mathrm{~N}$ & $\%$ & \\
\hline Delay & & & & & & & & & & & & & $<.01$ \\
\hline Less than 1 month & 97130 & 22.44 & 77821 & 23.32 & 4751 & 19.27 & 10867 & 19.06 & 2738 & 20.39 & 953 & 23.44 & \\
\hline 1-2 month & 230512 & 53.25 & 180573 & 54.11 & 12057 & 48.90 & 28580 & 50.12 & 7311 & 54.45 & 1991 & 48.98 & \\
\hline 2-3 month & 73203 & 16.91 & 53670 & 16.08 & 5092 & 20.65 & 11314 & 19.84 & 2376 & 17.69 & 751 & 18.47 & \\
\hline Beyond 3 months & 32038 & 7.40 & 21649 & 6.49 & 2757 & 11.18 & 6259 & 10.98 & 1003 & 7.47 & 370 & 9.10 & \\
\hline Age group, years & & & & & & & & & & & & & $<.01$ \\
\hline $18-49$ & 120908 & 27.93 & 88425 & 26.50 & 9193 & 37.28 & 16962 & 29.75 & 4993 & 37.18 & 1335 & 32.84 & \\
\hline $50-59$ & 149204 & 34.47 & 113863 & 34.12 & 8444 & 34.25 & 20749 & 36.39 & 4648 & 34.61 & 1500 & 36.90 & \\
\hline $60-69$ & 115556 & 26.69 & 92396 & 27.69 & 5252 & 21.30 & 14137 & 24.79 & 2852 & 21.24 & 919 & 22.61 & \\
\hline 70-79 & 41873 & 9.67 & 34540 & 10.35 & 1581 & 6.41 & 4613 & 8.09 & 860 & 6.40 & 279 & 6.86 & \\
\hline $80+$ & 5342 & 1.23 & 4489 & 1.35 & 187 & 0.76 & 559 & 0.98 & 75 & 0.56 & 32 & 0.79 & \\
\hline Insurance status & & & & & & & & & & & & & $<.01$ \\
\hline Uninsured & 10288 & 2.38 & 5214 & 1.56 & 2336 & 9.47 & 2080 & 3.65 & 508 & 3.78 & 150 & 3.69 & \\
\hline Medicaid & 32284 & 7.46 & 17014 & 5.10 & 5122 & 20.77 & 7905 & 13.86 & 1724 & 12.84 & 519 & 12.77 & \\
\hline $\begin{array}{l}\text { Medicare for } \\
\text { patients age 18-64 } \\
\text { years }\end{array}$ & 18427 & 4.26 & 12556 & 3.76 & 1092 & 4.43 & 4355 & 7.64 & 260 & 1.94 & 164 & 4.03 & \\
\hline $\begin{array}{l}\text { Medicare for } \\
\text { patients age } 65 \\
\text { years and older }\end{array}$ & 78393 & 18.11 & 65342 & 19.58 & 2701 & 10.95 & 8504 & 14.91 & 1354 & 10.08 & 492 & 12.10 & \\
\hline Private & 282360 & 65.23 & 225792 & 67.66 & 12533 & 50.83 & 32469 & 56.94 & 9180 & 68.36 & 2386 & 58.70 & \\
\hline Other/Unknown & 11131 & 2.57 & 7795 & 2.34 & 873 & 3.54 & 1707 & 2.99 & 402 & 2.99 & 354 & 8.71 & \\
\hline Region & & & & & & & & & & & & & $<.01$ \\
\hline Northeast & 88282 & 20.39 & 69648 & 20.87 & 5209 & 21.13 & 9451 & 16.57 & 3228 & 24.04 & 716 & 17.61 & \\
\hline Midwest & 113188 & 26.15 & 96532 & 28.93 & 2555 & 10.36 & 11552 & 20.26 & 1846 & 13.75 & 703 & 17.29 & \\
\hline South & 167020 & 38.58 & 119770 & 35.89 & 9531 & 38.65 & 33496 & 58.74 & 2771 & 20.64 & 1452 & 35.72 & \\
\hline West & 64423 & 14.88 & 47763 & 14.31 & 7362 & 29.86 & 2521 & 4.42 & 5583 & 41.58 & 1194 & 29.37 & \\
\hline Disease stage & & & & & & & & & & & & & $<.01$ \\
\hline I & 139090 & 32.13 & 109545 & 32.83 & 6986 & 28.33 & 17078 & 29.95 & 4309 & 32.09 & 1172 & 28.83 & \\
\hline II & 212337 & 49.05 & 161990 & 48.54 & 12545 & 50.88 & 28875 & 50.64 & 6817 & 50.77 & 2110 & 51.91 & \\
\hline III & 81456 & 18.82 & 62178 & 18.63 & 5126 & 20.79 & 11067 & 19.41 & 2302 & 17.14 & 783 & 19.26 & \\
\hline Hormone status & & & & & & & & & & & & & $<.01$ \\
\hline Negative & 129426 & 29.90 & 289044 & 86.61 & 7138 & 28.95 & 24592 & 43.13 & 3658 & 27.24 & 1138 & 28.00 & \\
\hline +ve/Borerline & 302546 & 69.89 & 240163 & 71.97 & 17425 & 70.67 & 32293 & 56.63 & 9744 & 72.56 & 2921 & 71.86 & \\
\hline No test & 911 & 0.21 & 650 & 0.19 & 94 & 0.38 & 135 & 0.24 & 26 & 0.19 & 6 & 0.15 & \\
\hline $\begin{array}{l}\text { Charlson-Deyo } \\
\text { score }\end{array}$ & & & & & & & & & & & & & $<.01$ \\
\hline 0 & 370017 & 85.48 & 289044 & 86.61 & 20816 & 84.42 & 44936 & 78.81 & 11846 & 88.22 & 3375 & 83.03 & \\
\hline 1 & 52024 & 12.02 & 37144 & 11.13 & 3252 & 13.19 & 9674 & 16.97 & 1393 & 10.37 & 561 & 13.80 & \\
\hline$\geq 2$ & 10842 & 2.50 & 7525 & 2.25 & 589 & 2.39 & 2410 & 4.23 & 189 & 1.41 & 129 & 3.17 & \\
\hline Income & & & & & & & & & & & & & $<.01$ \\
\hline
\end{tabular}




\begin{tabular}{|c|c|c|c|c|c|c|c|c|c|c|c|c|c|}
\hline Less than $\$ 38,000$ & 68777 & 15.89 & 39483 & 11.83 & 5906 & 23.95 & 21950 & 38.50 & 707 & 5.27 & 731 & 17.98 & \\
\hline$\$ 38,000-\$ 47,999$ & 93594 & 21.62 & 72768 & 21.81 & 5676 & 23.02 & 12856 & 22.55 & 1590 & 11.84 & 704 & 17.32 & \\
\hline$\$ 48,000-\$ 62,999$ & 115685 & 26.72 & 92629 & 27.76 & 6843 & 27.75 & 11894 & 20.86 & 3270 & 24.35 & 1049 & 25.81 & \\
\hline$\$ 63,000+$ & 154827 & 35.77 & 128833 & 38.61 & 6232 & 25.27 & 10320 & 18.10 & 7861 & 58.54 & 1581 & 38.89 & \\
\hline Education & & & & & & & & & & & & & $<.01$ \\
\hline $21 \%$ or more & 67019 & 15.48 & 35952 & 10.77 & 11130 & 45.14 & 17017 & 29.84 & 2189 & 16.30 & 731 & 17.98 & \\
\hline $13 \%-20 \%$ & 105698 & 24.42 & 75582 & 22.65 & 5729 & 23.23 & 20782 & 36.45 & 2613 & 19.46 & 992 & 24.40 & \\
\hline $7 \%-12 \%$ & 143104 & 33.06 & 118994 & 35.66 & 4965 & 20.14 & 13611 & 23.87 & 4287 & 31.93 & 1247 & 30.68 & \\
\hline Less than $7 \%$ & 117062 & 27.04 & 103185 & 30.92 & 2833 & 11.49 & 5610 & 9.84 & 4339 & 32.31 & 1095 & 26.94 & \\
\hline Facility Category & & & & & & & & & & & & & $<.01$ \\
\hline $\begin{array}{l}\text { Community Cancer } \\
\text { program }\end{array}$ & 41914 & 9.68 & 33681 & 10.09 & 2259 & 9.16 & 4381 & 7.68 & 1139 & 8.48 & 454 & 11.17 & \\
\hline $\begin{array}{l}\text { Comprehensive } \\
\text { community cancer } \\
\text { program }\end{array}$ & 195456 & 45.15 & 158222 & 47.41 & 9042 & 36.67 & 21754 & 38.15 & 4885 & 36.38 & 1553 & 38.20 & \\
\hline $\begin{array}{l}\text { Academic/research } \\
\text { program }\end{array}$ & 133333 & 30.80 & 94029 & 28.18 & 9816 & 39.81 & 22150 & 38.85 & 5809 & 43.26 & 1529 & 37.61 & \\
\hline $\begin{array}{l}\text { Other specified } \\
\text { types of cancer } \\
\text { programs }\end{array}$ & 62180 & 14.36 & 47781 & 14.32 & 3540 & 14.36 & 8735 & 15.32 & 1595 & 11.88 & 529 & 13.01 & \\
\hline Urban setting & & & & & & & & & & & & & $<.01$ \\
\hline Large Metropolitan & 233291 & 53.89 & 165986 & 49.74 & 17563 & 71.23 & 37170 & 65.19 & 10331 & 76.94 & 55.13 & 233291 & \\
\hline Small Metropolitan & 136317 & 31.49 & 111520 & 33.42 & 6015 & 24.39 & 14880 & 26.10 & 2792 & 20.79 & 1110 & 27.31 & \\
\hline Suburban & 46202 & 10.67 & 40793 & 12.22 & 831 & 3.37 & 3846 & 6.75 & 259 & 1.93 & 473 & 11.64 & \\
\hline Rural & 17073 & 3.94 & 15414 & 4.62 & 248 & 1.01 & 1124 & 1.97 & 46 & 0.34 & 241 & 5.93 & \\
\hline Year of Diagnosis & & & & & & & & & & & & & $<0.1$ \\
\hline 2004 & 27646 & 6.39 & 22439 & 6.72 & 1241 & 5.03 & 3124 & 5.48 & 661 & 4.92 & 181 & 4.45 & \\
\hline 2005 & 28221 & 6.52 & 22756 & 6.82 & 1353 & 5.49 & 3257 & 5.71 & 656 & 4.89 & 199 & 4.90 & \\
\hline 2006 & 30210 & 6.98 & 24243 & 7.26 & 1414 & 5.73 & 3573 & 6.27 & 738 & 5.50 & 242 & 5.95 & \\
\hline 2007 & 31452 & 7.27 & 24910 & 7.46 & 1640 & 6.65 & 3810 & 6.68 & 809 & 6.02 & 283 & 6.96 & \\
\hline 2008 & 31970 & 7.39 & 25028 & 7.50 & 1749 & 7.09 & 4044 & 7.09 & 858 & 6.39 & 291 & 7.16 & \\
\hline 2009 & 33939 & 7.84 & 26507 & 7.94 & 1813 & 7.35 & 4433 & 7.77 & 880 & 6.55 & 306 & 7.53 & \\
\hline 2010 & 34291 & 7.92 & 26369 & 7.90 & 1895 & 7.69 & 4646 & 8.15 & 1079 & 8.04 & 302 & 7.43 & \\
\hline 2011 & 36109 & 8.34 & 27583 & 8.27 & 2057 & 8.34 & 4915 & 8.62 & 1212 & 9.03 & 342 & 8.41 & \\
\hline 2012 & 36246 & 8.37 & 27518 & 8.25 & 2195 & 8.90 & 5004 & 8.78 & 1179 & 8.78 & 350 & 8.61 & \\
\hline 2013 & 37606 & 8.69 & 28334 & 8.49 & 2356 & 9.56 & 5207 & 9.13 & 1305 & 9.72 & 404 & 9.94 & \\
\hline 2014 & 36586 & 8.45 & 27362 & 8.20 & 2259 & 9.16 & 5234 & 9.18 & 1347 & 10.03 & 384 & 9.45 & \\
\hline 2015 & 35364 & 8.17 & 26212 & 7.85 & 2418 & 9.81 & 5010 & 8.79 & 1330 & 9.90 & 394 & 9.69 & \\
\hline 2016 & 33243 & 7.68 & 24452 & 7.33 & 2267 & 9.19 & 4763 & 8.35 & 1374 & 10.23 & 387 & 9.52 & \\
\hline \multicolumn{14}{|l|}{$\begin{array}{l}\text { Distance to } \\
\text { reporting facility, } \\
\text { miles }\end{array}$} \\
\hline Mean & 21.49 & & 22.92 & & 17.43 & & 15.16 & & 16.85 & & 32.57 & & \\
\hline SD & 80.91 & & 82.60 & & 75.64 & & 56.49 & & 105.73 & & 139.84 & & \\
\hline Median & 9.20 & & 10.10 & & 7.10 & & 7.00 & & 7.00 & & 10.10 & & \\
\hline
\end{tabular}


No. of days from

surgery to

chemotherapy

\begin{tabular}{lllllll} 
Mean & 48.73 & 47.38 & 54.22 & 53.92 & 49.74 & 50.18 \\
SD & 29.56 & 28.04 & 34.71 & 34.28 & 29.82 & 32.62 \\
\hline Median & 42.00 & 42.00 & 46.00 & 46.00 & 43.00 & 43.00
\end{tabular}

Table 2 demonstrates multivariable risk ratios for 30,60, 90, and 120 day delays based on the characteristics of Table 1: patient demographics, age, area-level SES, insurance status, and facility-level characteristics. The non-hispanic African American patients had the highest risk of mortality compared to non-hispanic whites as the number of delay days for each group increased. Older ages were associated with increased risk of mortality across all delay groups. Patients with a more advanced cancer stage had an increased mortality risk at each stage relative to stage one cancer in the 30-day delay group. 


\begin{tabular}{|c|c|c|c|c|c|c|c|c|}
\hline \multirow[b]{2}{*}{ Factor } & \multicolumn{2}{|c|}{ 30-Day Delay } & \multicolumn{2}{|c|}{ 60-Day Delay } & \multicolumn{2}{|c|}{ 90-Day Delay } & \multicolumn{2}{|c|}{ 120-Day Delay } \\
\hline & $\mathrm{RR}$ & $95 \% \mathrm{Cl}$ & $\mathrm{RR}$ & $95 \% \mathrm{Cl}$ & $\mathrm{RR}$ & $95 \% \mathrm{Cl}$ & $\mathrm{RR}$ & $95 \% \mathrm{Cl}$ \\
\hline \multicolumn{9}{|l|}{ Race } \\
\hline White & 1.00 & & 1.00 & & 1.00 & & 1.00 & \\
\hline Hispanic & 1.15 & $(1.11-1.20)$ & 1.33 & $(1.29-1.37)$ & 1.38 & $(1.32-1.44)$ & 1.47 & $(1.37-1.58)$ \\
\hline Black & 1.29 & $(1.26-1.32)$ & 1.41 & $(1.38-1.44)$ & 1.55 & $(1.50-1.60)$ & 1.69 & $(1.61-1.78)$ \\
\hline Asian & 1.14 & $(1.09-1.19)$ & 1.06 & $(1.02-1.11)$ & 1.02 & $(0.95-1.08)$ & 1.01 & $(0.91-1.13)$ \\
\hline Other/Unknown & 0.97 & $(0.90-1.04)$ & 1.22 & $(1.14-1.31)$ & 1.31 & $(1.18-1.46)$ & 1.37 & $(1.16-1.62)$ \\
\hline \multicolumn{9}{|l|}{ Age, years } \\
\hline $18-49$ & 1.00 & & 1.00 & & 1.00 & & 1.00 & \\
\hline $50-59$ & 1.13 & $(1.11-1.15)$ & 1.09 & $(1.07-1.11)$ & 1.05 & $(1.02-1.08)$ & 1.02 & $(0.97-1.07)$ \\
\hline $60-69$ & 1.31 & $(1.28-1.34)$ & 1.21 & $(1.19-1.24)$ & 1.16 & $(1.12-1.20)$ & 1.13 & $(1.07-1.19)$ \\
\hline $70-79$ & 1.48 & $(1.43-1.54)$ & 1.37 & $(1.33-1.42)$ & 1.34 & $(1.27-1.42)$ & 1.37 & $(1.26-1.49)$ \\
\hline $80-99$ & 1.46 & $(1.36-1.57)$ & 1.61 & $(1.50-1.72)$ & 1.67 & $(1.51-1.84)$ & 1.90 & $(1.64-2.20)$ \\
\hline \multicolumn{9}{|l|}{ Insurance status } \\
\hline Private & 1.00 & & 1.00 & & 1.00 & & 1.00 & \\
\hline Uninsured & 1.36 & $(1.29-1.43)$ & 1.43 & $(1.37-1.50)$ & 1.50 & $(1.41-1.60)$ & 1.72 & $(1.57-1.90)$ \\
\hline Medicaid & 1.30 & $(1.26-1.34)$ & 1.52 & $(1.48-1.56)$ & 1.67 & $(1.60-1.73)$ & 1.85 & $(1.74-1.96)$ \\
\hline Medicare for patients age 18-64 years & 1.25 & $(1.20-1.30)$ & 1.37 & $(1.32-1.42)$ & 1.51 & $(1.44-1.59)$ & 1.67 & $(1.54-1.80)$ \\
\hline Medicare for patients age 65 years and older & 1.11 & $(1.08-1.14)$ & 1.10 & $(1.07-1.13)$ & 1.12 & $(1.07-1.16)$ & 1.16 & $(1.08-1.24)$ \\
\hline Other/unknown & 0.77 & $(0.74-0.80)$ & 1.07 & $(1.03-1.12)$ & 1.23 & $(1.15-1.32)$ & 1.26 & $(1.13-1.41)$ \\
\hline \multicolumn{9}{|l|}{ Region } \\
\hline South & 1.00 & & 1.00 & & 1.00 & & 1.00 & \\
\hline Northeast & 1.33 & $(1.30-1.36)$ & 1.22 & $(1.20-1.25)$ & 1.23 & $(1.20-1.27)$ & 1.21 & $(1.16-1.27)$ \\
\hline Midwest & 0.99 & $(0.98-1.02)$ & 0.87 & $(0.86-0.89)$ & 0.82 & $(0.80-0.85)$ & 0.79 & $(0.75-0.83)$ \\
\hline West & 1.19 & $(1.16-1.22)$ & 1.09 & $(1.07-1.12)$ & 1.12 & $(1.08-1.16)$ & 1.17 & $(1.11-1.24)$ \\
\hline \multicolumn{9}{|l|}{ Stage } \\
\hline III & 1.00 & & 1.00 & & 1.00 & & 1.00 & \\
\hline II & 1.31 & $(1.29-1.34)$ & 1.11 & $(1.09-1.13)$ & 0.99 & $(0.96-1.03)$ & 0.90 & $(0.86-0.95)$ \\
\hline I & 1.61 & $(1.57-1.64)$ & 1.13 & $(1.27-1.32)$ & 1.16 & $(1.13-1.20)$ & 1.10 & $(1.04-1.16)$ \\
\hline \multicolumn{9}{|l|}{ Hormone status } \\
\hline Negative & 1.00 & & 1.00 & & 1.00 & & 1.00 & \\
\hline Positive & 1.55 & $(1.52-1.57)$ & 1.46 & $(1.44-1.49)$ & 1.45 & $(1.41-1.49)$ & 1.45 & $(1.39-1.51)$ \\
\hline Unknown & 1.17 & $(1.00-1.35)$ & 1.75 & $(1.52-2.02)$ & 1.80 & $(1.47-2.21)$ & 1.97 & $(1.46-2.65)$ \\
\hline \multicolumn{9}{|l|}{ Charlson-Deyo score } \\
\hline 0 & 1.00 & & 1.00 & & 1.00 & & 1.00 & \\
\hline 1 & 1.18 & $(1.15-1.20)$ & 1.12 & $(1.10-1.14)$ & 1.13 & $(1.10-1.17)$ & 1.12 & $(1.06-1.18)$ \\
\hline$\geq 2$ & 1.30 & $(1.24-1.37)$ & 1.34 & $(1.28-1.39)$ & 1.39 & $(1.31-1.48)$ & 1.39 & $(1.26-1.53)$ \\
\hline
\end{tabular}




\begin{tabular}{|c|c|c|c|c|c|c|c|c|}
\hline \multirow[b]{2}{*}{ Factor } & \multicolumn{2}{|c|}{ 30-Day Delay } & \multicolumn{2}{|c|}{ 60-Day Delay } & \multicolumn{2}{|c|}{ 90-Day Delay } & \multicolumn{2}{|c|}{ 120-Day Delay } \\
\hline & $\mathrm{RR}$ & $95 \% \mathrm{Cl}$ & $\mathrm{RR}$ & $95 \% \mathrm{Cl}$ & $\mathrm{RR}$ & $95 \% \mathrm{Cl}$ & $\mathrm{RR}$ & $95 \% \mathrm{Cl}$ \\
\hline \multicolumn{9}{|l|}{ Income } \\
\hline$\$ 63,000+$ & 1.00 & & 1.00 & & 1.00 & & 1.00 & \\
\hline$\$ 48,000-\$ 62,999$ & 1.03 & $(1.00-1.05)$ & 1.01 & $(0.99-1.03)$ & 0.99 & $(0.96-1.03)$ & 0.97 & $(0.92-1.03)$ \\
\hline$\$ 38,000-\$ 47,999$ & 1.07 & $(1.04-1.09)$ & 1.04 & $(1.01-1.07)$ & 1.02 & $(0.98-1.06)$ & 0.98 & $(0.92-1.05)$ \\
\hline Less than $\$ 38,000$ & 1.13 & $(1.09-1.16)$ & 1.08 & $(1.04-1.11)$ & 1.06 & $(1.01-1.11)$ & 1.03 & $(0.96-1.12)$ \\
\hline \multicolumn{9}{|l|}{ Education } \\
\hline $21 \%$ or more & 1.00 & & 1.00 & & 1.00 & & 1.00 & \\
\hline $13 \%-20 \%$ & 0.97 & $(0.95-99)$ & 0.95 & $(0.93-0.97)$ & 0.95 & $(0.92-0.99)$ & 0.95 & $(0.90-1.01)$ \\
\hline $7 \%-12 \%$ & 0.95 & $(0.92-0.97)$ & 0.91 & $(0.88-0.93)$ & 0.87 & $(0.84-0.91)$ & 0.88 & $(0.82-0.94)$ \\
\hline Less than $7 \%$ & 0.85 & $(0.83-0.88)$ & 0.80 & $(0.77-0.82)$ & 0.74 & $(0.70-0.78)$ & 0.72 & $(0.66-0.77)$ \\
\hline \multicolumn{9}{|l|}{ Facility Category } \\
\hline Community Cancer Care & 1.00 & & 1.00 & & 1.00 & & 1.00 & \\
\hline Comprehensive community cancer program & 0.91 & $(0.89-0.93)$ & 0.88 & $(0.86-0.90)$ & 0.83 & $(0.80-0.87)$ & 0.80 & $(0.75-0.86)$ \\
\hline Academic/research program & 1.11 & $(1.08-1.14)$ & 1.11 & $(1.08-1.14)$ & 1.07 & $(1.03-1.12)$ & 1.05 & $(0.99-1.12)$ \\
\hline Other specified types of cancer programs & 1.00 & $(0.97-1.03)$ & 0.96 & $(0.93-0.99)$ & 0.92 & $(0.88-0.96)$ & 0.89 & $(0.82-0.96)$ \\
\hline \multicolumn{9}{|l|}{ Urban Setting } \\
\hline Large metropolitan & 1.00 & & 1.00 & & 1.00 & & 1.00 & \\
\hline Small metropolitan & 0.98 & $(0.96-0.99)$ & 0.88 & $(0.86-0.89)$ & 0.83 & $(0.81-0.86)$ & 0.76 & $(0.73-0.79)$ \\
\hline Suburban & 1.01 & $(0.99-1.04)$ & 0.92 & $(0.90-0.95)$ & 0.83 & $(0.80-0.87)$ & 0.75 & $(0.70-0.81)$ \\
\hline Rural & 1.03 & $(0.99-1.08)$ & 0.90 & $(0.86-0.93)$ & 0.77 & $(0.73-0.83)$ & 0.74 & $(0.67-0.83)$ \\
\hline \multicolumn{9}{|l|}{ Year of diagnosis } \\
\hline 2004 & 1.00 & & 1.00 & & 1.00 & & 1.00 & \\
\hline 2005 & 1.04 & $(0.99-1.08)$ & 1.04 & $(1.00-1.09)$ & 1.04 & $(0.97-1.10)$ & 1.09 & $(0.99-1.20)$ \\
\hline 2006 & 1.12 & $(1.08-1.16)$ & 1.06 & $(1.02-1.10)$ & 1.10 & $(1.04-1.17)$ & 1.13 & $(1.03-1.24)$ \\
\hline 2007 & 1.16 & $(1.12-1.21)$ & 1.12 & $(1.08-1.16)$ & 1.09 & $(1.02-1.15)$ & 1.10 & $(1.01-1.21)$ \\
\hline 2008 & 1.26 & $(1.21-1.31)$ & 1.13 & $(1.09-1.17)$ & 1.06 & $(0.99-1.12)$ & 1.07 & $(0.98-1.18)$ \\
\hline 2009 & 1.32 & $(1.27-1.37)$ & 1.08 & $(1.04-1.12)$ & 1.04 & $(0.98-1.10)$ & 1.02 & $(0.93-0.85)$ \\
\hline 2010 & 1.31 & $(1.26-1.36)$ & 1.02 & $(0.98-1.06)$ & 0.94 & $(0.88-0.99)$ & 0.93 & $(0.85-1.02)$ \\
\hline 2011 & 1.19 & $(1.15-1.24)$ & 0.91 & $(0.88-0.95)$ & 0.87 & $(0.82-0.92)$ & 0.84 & $(0.76-0.92)$ \\
\hline 2012 & 1.21 & $(1.17-1.26)$ & 0.91 & $(0.88-0.95)$ & 0.81 & $(0.76-0.86)$ & 0.72 & $(0.65-0.79)$ \\
\hline 2013 & 1.21 & $(1.16-1.25)$ & 0.90 & $(0.87-0.94)$ & 0.80 & $(0.76-0.85)$ & 0.75 & $(0.68-0.82)$ \\
\hline 2014 & 1.12 & $(1.08-1.16)$ & 0.80 & $(0.77-0.83)$ & 0.72 & $(0.68-0.77)$ & 0.67 & $(0.61-0.74)$ \\
\hline 2015 & 1.15 & $(1.11-1.20)$ & 0.80 & $(0.77-0.84)$ & 0.71 & $(0.67-0.75)$ & 0.63 & $(0.57-0.70)$ \\
\hline 2016 & 1.12 & $(1.08-1.17)$ & 0.83 & $(0.80-0.86)$ & 0.76 & $(0.71-0.80)$ & 0.70 & $(0.63-0.77)$ \\
\hline
\end{tabular}

Insurance type was associated with survival outcomes among patients with later delays. Patients who were uninsured or who were enrolled in Medicaid or Medicare had an increased risk of mortality in the 60,90 , and 120 day delay groups. Notably, this trend was not consistent with the 
privately insured 30 day delay group who showed a decreased risk of mortality. The facility type was associated with a decreased risk of mortality across all delay groups. The only increase in risk relative to the community cancer care was found within the academic research programs. The hormone receptor-positive status was associated with a higher risk of mortality across the delay groups relative to hormone receptor-negative (1.55, $1.46,1.45,1.45)$. For the Charlson Deyo score, each of the varying day groups had the highest risk relative to the Charlson Deyo score of 2.

Table 3 demonstrates Cox Hazard ratios that do not change over time to assess the impact of treatment delay post surgery on mortality. In comparison to delayment of treatment, there was an increase in associated risk found among all studied groups Those with medicare for both age groups had a decreased associated risk relative to privately insured individuals, whereas those without insurance and had medicaid had a decreased survival rate.

\section{Discussion}

The study determined the suggested effect of delayed adjuvant chemotherapy for breast cancer treatment among five groups. The findings were consonant with previous research that used varied locations and samples. Prior studies have indicated a higher risk of mortality among women receiving adjuvant chemotherapy with increased days of delay after surgery [44]. This suggests clinical and nonclinical factors as important and providers should avoid delays.

Low socioeconomic status and lack of health insurance coverage combined have been cited as a predictor of delay in many previous studies [45]. These results were replicated using the California Cancer Registry database [46]. Similar to our findings, the young women patients of lower income and a longer delay treatment have a significantly increased risk of mortality compared with those with a shorter number of delays. This adverse impact on survival was more pronounced in African American women, those with public or no insurance.

The study found Black and Hispanic women and women without private insurance were more likely than white women with private insurance to be at risk. This suggests these women with public or no insurance compared with those with private insurance and women with low socioeconomic status compared with those with high socioeconomic status were shown to have longer initiated delay of treatment [47]. This suggests the need to initiate and diagnose prior to the issue exacerbating which can be addressed through more local options to get diagnosed, with or without insurance. As an example, it has suggested that a mobile mammogram has been an effective measure for addressing racial diversity and resulting in a higher recall rate [48].

It is well supported that areas with lower socioeconomic status have decreased opportunities to get education [49]. Decreased education and access to quality facilities has been associated with a higher risk of mortality for those affected by breast cancer [50]. Women with higher education levels attend breast cancer screening to a greater extent than less educated women [51] [52].

The findings on delay in breast cancer diagnosis and treatment suggest decreased quality of care among African American and Hispanic patients. A patient's environment such as a rural setting may be less likely to have specialized equipment or trained personnel. For treatment to be effective, there must be attention for how the provider reaches out to patients post-treatment [53] [54]. Whether for screening or diagnostic purposes, many studies' results suggest the health system must support training for following up with patient effective communication and providing feedback [55] This supports this paper's findings similarly suggesting different regions' facilities contribute to a decreased ability to go in for screenings and thus delay treatment.

The findings on delay in breast cancer diagnosis and treatment suggest Black and Hispanic groups had decreased access to quality of care, services, and education. Quality of care relates to distrust. Previous studies have assessed distrust and adjuvant cancer treatment with increased risk of mortality due to delayed adjuvant treatment for breast cancer among patients [56] [57] [58]. The study suggests since there is a difference in allocating resources and communities for White patients when treating the disease, they can more easily recover because they trust their provider about the benefits of chemotherapy. This suggests the need to include ways to educate providers about assessing medical distrust among different communities as a way to promote cultural competence.

The study's interpretations are limited by the given design of the National Cancer Database. The NCDB provides a holistic, comprehensive picture for its samples, including a number of less commonly available details that enable subtle cultural differences among each group to be studied. NCDB is a comprehensive source, approximately $70 \%$ of newly diagnosed cancer cases in the United States reported [59] and 34 million cases reported, however, it does not qualify as population-based because if a patient happens to choose a different hospital, one that is not an NCDB participant, his or her data are not included Data. This data additionally was limited to the time span of 2004 to 2016 . Since NCDB is a hospital-based registry, findings may not be generalizable to all patients in the United States. Analysis of a study from a population-based data source in comparison to the findings presented could allow for further analysis of delayed treatment of breast cancer.

\section{Declarations}

\section{Funding}


No funding or support was used for this study. The study was performed using UVA Cancer Center resources

\section{Conflicts of interest}

All authors declare that they have no conflicts of interest.

\section{Author Contributions}

Conceptualization: Desai and Balkrishnan Data and Analysis: Desai and Balkrishnan Paper Writing Desai, Narayan and Hsiao Critical Revision: All authors

\section{Ethics approval}

All procedures performed in studies involving human participants were in accordance with the ethical standards of the institutional and/or national research committee and with the 1964 Helsinki declaration and its later amendments or comparable ethical standards.

The study protocol was exempted by the UVA IRB

\section{Consent to participate}

The study uses an extant database. All participants consented for their data to be collected.

Consent for publication All authors have reviewed the manuscript and consented to its submission for publication.

\section{References}

1. Momenimovahed Z, Salehiniya H (2019) Epidemiological characteristics of and risk factors for breast cancer in the world. Breast Cancer: Targets Therapy 11:151-164. doi:10.2147/bctt.s176070

2. Desantis CE, Ma J, Gaudet MM et al (2019) Breast cancer statistics, CA: A Cancer Journal for Clinicians. 2019;69(6):438-451. doi: $10.3322 /$ caac. 21583

3. Hortobagyi GN, de la Garza Salazar J, Pritchard K et al (2005) ABREAST Investigators The global breast cancer burden: variations in epidemiology and survival. Clinical Breast Cancer 6(5):391-401

4. Parkin DM, Fernández LM (2006) Use of statistics to assess the global burden of breast cancer. Breast J 12(s1):S70-S80

5. Desantis CE, Ma J, Goding Sauer A, Newman LA, Jemal A (2017) Breast cancer statistics, 2017, racial disparity in mortality by state. CA Cancer J Clin 67(6):439-448

6. Zaidi Z, Dib HA (2019) Abstract 4191: The worldwide female breast cancer incidence and survival, 2018. Epidemiology. doi:10.1158/15387445.sabcs18-4191

7. DeSantis C, Ma J, Bryan L, Jemal A (2014) Breast cancer statistics, 2013. CA Cancer J Clin 64(1):52-62

8. Waks AG, Winer EP (2019) Breast Cancer Treatment: A Review. JAMA 321(3):288-300. doi:10.1001/jama.2018.19323

9. Mariotto AB, Etzioni R, Hurlbert M et al (2017) Estimation of the number of women living with breast cancer in the United States. Cancer Epidemiol Biomarkers Prev 26(6):809-815. doi:10.1158/1055-9965.EPI-16-0889

10. Maibenco DC, Dombi GW, Kau TY, Severson RK (2006) Significance of micrometastases on the survival of women with T1 breast cancer. Cancer 107(6):1234-1239. doi:10.1002/cncr.22112

11. Boér K (2010) A gyógyszeres kezelés szerepe a korai emlôrák kezelésében [Adjuvant chemotherapy of early stage breast cancer]. Orv Hetil 151(9):344-353. doi:10.1556/OH.2010.28824

12. Andersson Y, Bergkvist L, Frisell J, Boniface JD (2018) Long-term breast cancer survival in relation to the metastatic tumor burden in axillary lymph nodes. Breast Cancer Res Treat 171(2):359-369. doi:10.1007/s10549-018-4820-0

13. Early Breast Cancer Trialists' Collaborative Group (EBCTCG), Peto R, Davies C et al. Comparisons between different polychemotherapy regimens for early breast cancer: meta-analyses of long-term outcome among 100,000 women in 123 randomised trials. Lancet. 2012;379(9814):432444. doi:10.1016/S0140-6736(11)61625-5

14. Rossi L, Stevens D, Pierga J-Y et al. Impact of Adjuvant Chemotherapy on Breast Cancer Survival: A Real-World Population. Plos One. 2015;10(7). doi:10.1371/journal.pone.0132853

15. Zhan Q-H, Fu J-Q, Fu F-M, Zhang J, Wang C (2017) Survival and time to initiation of adjuvant chemotherapy among breast cancer patients: a systematic review and meta-analysis. Oncotarget 9(2):2739-2751. doi:10.18632/oncotarget.23086

16. Biagi JJ, Raphael MJ, Mackillop WJ, Kong W, King WD, Booth CM (2011) Association between time to initiation of adjuvant chemotherapy and survival in colorectal cancer: a systematic review and meta-analysis. JAMA 305(22):2335-2342 
17. Gao P, Huang X-Z, Song Y-X et al. Impact of timing of adjuvant chemotherapy on survival in stage III colon cancer: a population-based study. BMC Cancer. 2018;18(1). doi:10.1186/s12885-018-4138-7

18. Sen F, Pilanci KN (2016) Adjuvant Systemic Therapy in Stage II and III Colon Cancer. Colorectal Cancer - From Pathogenesis to Treatment. doi:10.5772/63208

19. Salner AL, Smith S, Yu PP (2018) Timing of adjuvant chemotherapy administration for early breast cancer. J Clin Oncol 36(30_suppl):62-62. doi:10.1200/jco.2018.36.30_suppl.62

20. Bos A, Erning FV, Gestel YV, Creemers G, Punt C, Oijen MV, Lemmens V (2015) Timing of adjuvant chemotherapy and its relation to survival among patients with stage III colon cancer. Eur J Cancer 51(17):2553-2561. doi:10.1016/j.ejca.2015.08.016

21. Kennedy RD, Bylesjo M, Kerr P et al (2011) Development and independent validation of a prognostic assay for stage II colon cancer using formalin-fixed paraffin-embedded tissue. J Clin Oncol 29(35):4620-4626. doi:10.1200/JC0.2011.35.4498

22. Huang S-M, Chen Y-C, Chen W-Y et al (2019) Optimal Timing for Postsurgical Adjuvant Therapy in Patients with Gastric Cancer: A Propensity Score Matching Study. J Cancer 10(2):332-340. doi:10.7150/jca.27753

23. Ma SJ, Oladeru OT, Miccio JA, lovoli AJ, Hermann GM, Singh AK. Association of Timing of Adjuvant Therapy With Survival in Patients With Resected Stage I to II Pancreatic Cancer. JAMA Network Open. 2019;2(8). doi:10.1001/jamanetworkopen.2019.9126

24. Kim HW, Lee J-C, Lee J, Kim JW, Kim J, Hwang J-H. Early versus delayed initiation of adjuvant treatment for pancreatic cancer. Plos One. 2017;12(3). doi:10.1371/journal.pone.0173960

25. Mönig SP, Bonino MA (2019) Delay in initiation adjuvant S-1 monotherapy for gastric cancer: important prognostic factor. Translational Gastroenterology Hepatology 4:61-61. doi:10.21037/tgh.2019.07.03

26. Yu K-D, Fan L, Qiu L-X, Ling H, Jiang Y-Z, Shao Z-M (2016) Influence of delayed initiation of adjuvant chemotherapy on breast cancer survival is subtype-dependent. Oncotarget 8(28):46549-46556. doi:10.18632/oncotarget.10551

27. Farolfi A, Scarpi E, Rocca A et al (2015) Time to initiation of adjuvant chemotherapy in patients with rapidly proliferating early breast cancer. Eur J Cancer 51(14):1874-1881. doi:10.1016/j.ejca.2015.07.003

28. Pronzato P, Campora E, Amoroso D, Bertelli G, Botto F, Conte PF, Sertoli MR, Rosso R (1989) Impact of administration-related factors on outcome of adjuvant chemotherapy for primary breast cancer. Am J Clin Oncol 12(6):481-485. https://doi.org/10.1097/00000421-198912000-00004

29. Gagliato Dde M, Gonzalez-Angulo AM, Lei X, Theriault RL, Giordano SH, Valero V, Hortobagyi GN, Chavez-Macgregor M. Clinical impact of delaying initiation of adjuvant chemotherapy in patients with breast cancer. J Clin Oncol. 2014 Mar 10;32(8):735 - 44. doi:

10.1200/JC0.2013.49.7693. Epub 2014 Jan 27. PMID: 24470007; PMCID: PMC3940536

30. Downing A, Twelves C, Forman D, Lawrence G, Gilthorpe MS (2014) Time to begin adjuvant chemotherapy and survival in breast cancer patients: a retrospective observational study using latent class analysis. Breast J 20(1):29-36. doi:10.1111/tbj.12209

31. Lohrisch C, Paltiel C, Gelmon K et al (2006) Impact on survival of time from definitive surgery to initiation of adjuvant chemotherapy for earlystage breast cancer. J Clin Oncol 24(30):4888-4894. doi:10.1200/JC0.2005.01.6089

32. Chavez-MacGregor M, Clarke CA, Lichtensztajn DY, Giordano SH (2016) Delayed Initiation of Adjuvant Chemotherapy Among Patients With Breast Cancer. JAMA Oncol 2(3):322-329. doi:10.1001/jamaoncol.2015.3856

33. Hershman DL, Wang X, McBride R, Jacobson JS, Grann VR, Neugut Al (2006) Delay of adjuvant chemotherapy initiation following breast cancer surgery among elderly women. Breast Cancer Res Treat 99(3):313-321. doi:10.1007/s10549-006-9206-Z

34. Nurgalieva ZZ, Franzini L, Morgan RO, Vernon SW, Liu CC, Du XL (2013) Impact of timing of adjuvant chemotherapy initiation and completion after surgery on racial disparities in survival among women with breast cancer. Med Oncol 30(1):419. doi:10.1007/s12032-012-0419-1

35. Altundag M, Çelik I, Özişik Y (2000) Is there a range of time for initiation of adjuvant chemotherapy in patients with malignancy? Ann Oncol 11(9):1209. doi:10.1023/a:1008339804441

36. Desch CE, Mcniff KK, Schneider EC et al (2008) American Society of Clinical Oncology/National Comprehensive Cancer Network Quality Measures. J Clin Oncol 26(21):3631-3637. doi:10.1200/jco.2008.16.5068

37. Buzdar AU, Smith TL, Powell KC, Blumenschein GR, Gehan EA (1982) Effect of timing of initiation of adjuvant chemotherapy on disease-free survival in breast cancer. Breast Cancer Res Treat 2(2):163-169. doi:10.1007/bf01806452

38. Cold S, Düring M, Ewertz M, Knoop A, Møller S (2005) Does timing of adjuvant chemotherapy influence the prognosis after early breast cancer? Results of the Danish Breast Cancer Cooperative Group (DBCG). Br J Cancer 93(6):627-632. doi:10.1038/sj.bjc.6602734

39. Sánchez CJ, Ruiz A, Martín M et al (2006) Influence of Timing of Initiation of Adjuvant Chemotherapy Over Survival in Breast Cancer: A Negative Outcome Study by the Spanish Breast Cancer Research Group (GEICAM). Breast Cancer Res Treat 101(2):215-223. doi:10.1007/s10549-006-9282-0

40. Shannon C, Ashley S, Smith I (2003) Does Timing of Adjuvant Chemotherapy for Early Breast Cancer Influence Survival? J Clin Oncol 21(20):3792-3797. doi:10.1200/jco.2003.01.073

41. Gwyn K, Bondy ML, Cohen DS et al (2004) Racial differences in diagnosis, treatment, and clinical delays in a population-based study of patients with newly diagnosed breast carcinoma. Cancer 100(8):1595-1604. doi:10.1002/cncr.20169

Page 12/14 
42. Fedewa SA, Ward EM, Stewart AK, Edge SB (2010) Delays in adjuvant chemotherapy treatment among patients with breast cancer are more likely in African American and Hispanic populations: a national cohort study 2004-2006. J Clin Oncol 28(27):4135-4141.

doi:10.1200/JC0.2009.27.2427

43. Gorin SS, Heck JE, Cheng B, Smith SJ (2006) Delays in breast cancer diagnosis and treatment by racial/ethnic group. Arch Intern Med 166(20):2244-2252. doi:10.1001/archinte.166.20.2244

44. Fedewa SA, Ward EM, Stewart AK, Edge SB (2010) Delays in Adjuvant Chemotherapy Treatment Among Patients With Breast Cancer Are More Likely in African American and Hispanic Populations: A National Cohort Study 2004-2006. J Clin Oncol 28(27):4135-4141. doi:10.1200/jco.2009.27.2427

45. Lannin DR (1998) Influence of Socioeconomic and Cultural Factors on Racial Differences in Late-Stage Presentation of Breast Cancer. JAMA 279(22):1801. doi:10.1001/jama.279.22.1801

46. Smith EC, Ziogas A, Anton-Culver H (2013) Delay in Surgical Treatment and Survival After Breast Cancer Diagnosis in Young Women by Race/Ethnicity. JAMA Surgery 148(6):516. doi:10.1001/jamasurg.2013.1680

47. Berrian JL, Liu Y, Lian M, Schmaltz CL, Colditz GA. Relationship between insurance status and outcomes for patients with breast cancer in Missouri. Cancer Published online November 17, 2020. doi:10.1002/cncr.33330

48. Stanley E, Lewis MC, Irshad A et al (2017) Effectiveness of a Mobile Mammography Program. Am J Roentgenol 209(6):1426-1429. doi:10.2214/ajr.16.17670

49. Taheri M, Tavakol M;AkbariME, Almasi-Hashiani A, Abbasi M Relationship of Socio Economic Status, Income, and Education with the Survival Rate of Breast Cancer: A Meta-Analysis. Iranian journal of public health. 2019;48(8). Accessed November 17, 2020. https://pubmed.ncbi.nlm.nih.gov/32292725/

50. Ramachandran A, Freund KM, Bak SM, Heeren TC, Chen CA, Battaglia TA (2015) Multiple Barriers Delay Care Among Women with Abnormal Cancer Screening Despite Patient Navigation. Journal of Women's Health 24(1):30-36. doi:10.1089/jwh.2014.4869

51. Hussain SK, Altieri A, Sundquist J, Hemminki K (2007) Influence of education level on breast cancer risk and survival in Sweden between 1990 and 2004. Int J Cancer 122(1):165-169. doi:10.1002/ijc.23007

52. Wu T-Y, Lee J (2019) Promoting Breast Cancer Awareness and Screening Practices for Early Detection in Low-Resource Settings. European Journal of Breast Health 15(1):18-25. doi:10.5152/ejbh.2018.4305

53. Gorin SS, Ashford AR, Lantigua R et al (2006) Effectiveness of Academic Detailing on Breast Cancer Screening among Primary Care Physicians in an Underserved Community. The Journal of the American Board of Family Medicine 19(2):110-121. doi:10.3122/jabfm.19.2.110

54. Bastani R, Yabroff KR, Myers RE, Glenn B (2004) Interventions to improve follow-up of abnormal findings in cancer screening. Cancer 101(S5):1188-1200. doi:10.1002/cncr.20506

55. Ginsburg O, Yip C, Brooks A et al (2020) Breast cancer early detection: A phased approach to implementation. Cancer 126(S10):2379-2393. doi:10.1002/cncr.32887

56. Dean LT, Moss SL, McCarthy AM, Armstrong K (2017) Healthcare System Distrust, Physician Trust, and Patient Discordance with Adjuvant Breast Cancer Treatment Recommendations. Cancer Epidemiology Biomarkers Prevention 26(12):1745-1752. doi:10.1158/1055-9965.epi-1704791

57. Jassem J, Ozmen V, Bacanu F et al (2013) Delays in diagnosis and treatment of breast cancer: a multinational analysis. Eur J Pub Health 24(5):761-767. doi:10.1093/eurpub/ckt131

58. Heath J, Paulishak M, Kasales C, Schubart J, Kass R. Reasons symptomatic breast cancer patients delay seeking medical care. Journal of Unexplored Medical Data. 2019;2019. doi:10.20517/2572-8180.2018.011

59. National Cancer Database. American College of Surgeons. Published 2020. Accessed February 19 (2021) https://www.facs.org/quality\%20programs/cancer/ncdb

\section{Figures}




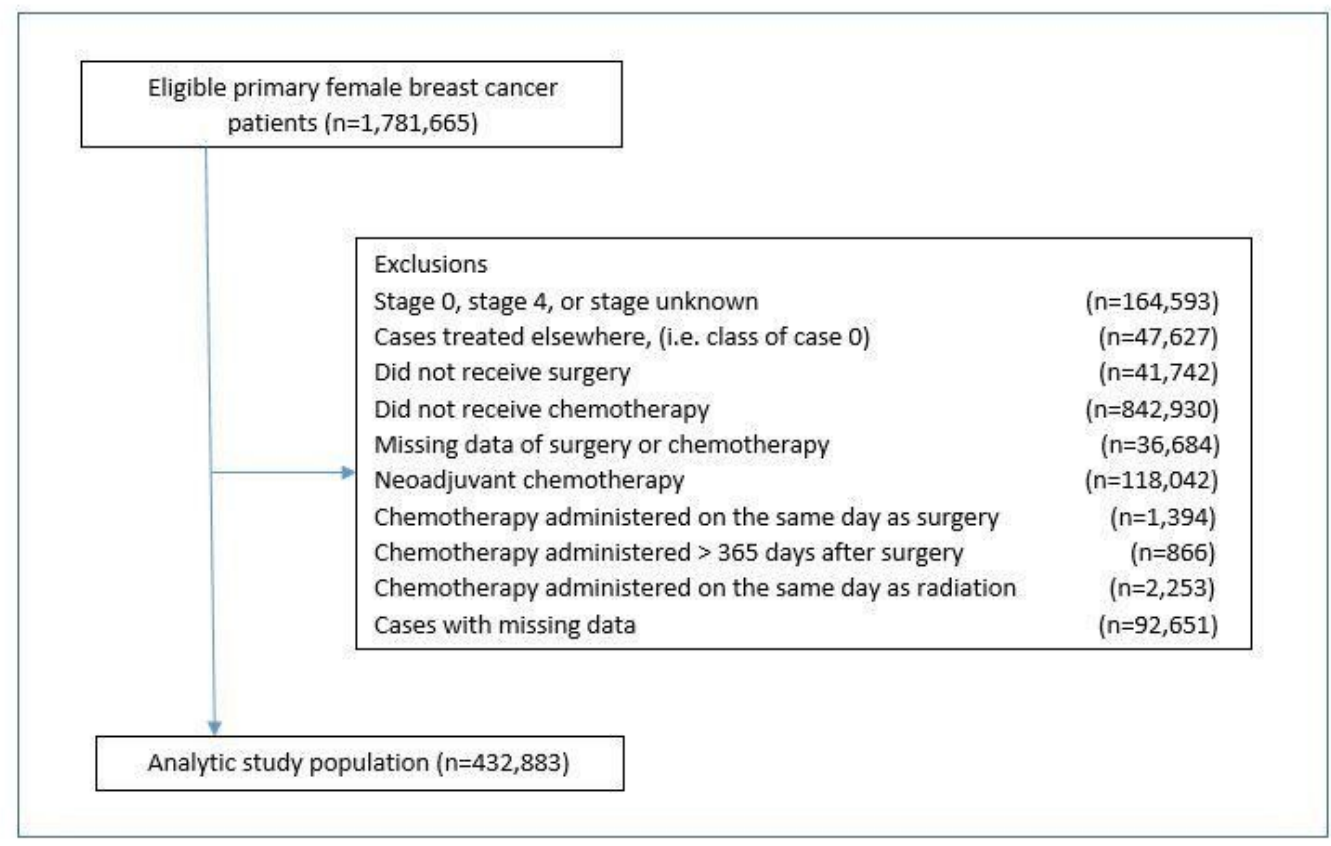

Figure 1

Derivation of the study population, National Cancer Database 2004 to 2016 